\title{
Se dresser contre l'injustice et travailler à la réconciliation dans le domaine des soins de santé
}

\author{
par Jody Ciufo, Zack Dumont, Tania Mysak, Shirin Abadi et Tamar Koleba
}

DOI: 10.4212/cjhp.v74i4.3204

Les récentes découvertes de tombes anonymes contenant des restes d'enfants sur le site d'anciens pensionnats autochtones ont profondément ébranlé les Canadiens et bien d'autres dans le monde. En tant que personnes, nous devons faire face à la violence et à l'injustice du colonialisme qui sévit dans ce pays et reconnaître le traumatisme intergénérationnel vécu par les survivants de ces pensionnats. Nous devons nous tenir aux côtés des peuples autochtones et prendre des mesures pour mettre un terme à la discrimination et à l'exploitation qui perdurent.

En tant quassociation professionnelle, comment la Société canadienne des pharmaciens d'hôpitaux (SCPH) peut-elle se dresser contre l'injustice et travailler en vue de la réconciliation dans le domaine des soins de santé? Ce travail commence par une reconnaissance de la complicité historique des systèmes de soins de santé canadiens, qui ont soumis des communautés autochtones, y compris des enfants dans les pensionnats, à des expériences et des tests médicaux contraires à la déontologie (https://academic.oup. com/pch/article/19/2/64/2647158) en matière d'interventions chirurgicales, d'administration de médicaments et de vaccins (https://www.cmaj.ca/content/193/11/E381).

Dans chaque province, l'horrible héritage du colonialisme se transmet aujourd'hui sous forme de racisme institutionnel dans notre système de soins de santé. En Saskatchewan, des femmes autochtones rapportent avoir été stérilisées de force pas plus tard qu'en 2019 (https:// globalnews.ca/news/7920118/indigenous-women-sterilization-senate-report/). En 2020, Joyce Echaquan est décédée dans un hôpital du Québec après avoir filmé les maltraitances à caractère raciste de la part du personnel. En Colombie-Britannique, le rapport In Plain Sight de 2020 dévoile que $84 \%$ des 9000 Autochtones participant à l'enquête avaient subi de la discrimination en accédant à des services de santé (https://engage.gov.bc.ca/app/uploads/ sites/613/2021/02/In-Plain-Sight-Data-Report_Dec2020. pdf1_.pdf). Le racisme dans les soins de santé est omniprésent et mortel.

En tant que corps de professionnels de la santé, la SCPH se rend compte des graves préjudices qu’a causé et que continue de causer - le système de soins de santé canadien aux peuples autochtones et aux personnes de couleur. Nous reconnaissons que la responsabilité de changer les systèmes et les institutions dans lesquels nous travaillons nous incombe. Le racisme qui sévit sur nos lieux de travail est inacceptable : les traumatismes, les maltraitances et les offenses doivent cesser.

Le conseil appelle les membres de la SCPH à se joindre à lui pour combattre le racisme systémique là où nous le rencontrons, qu'il se manifeste sous forme de traitement inéquitable des patients ou d'obstacles auxquels sont confrontés les pharmaciens membres des communautés de PANDC (personnes autochtones, noires et de couleur) pour faire progresser leur carrière. Ce travail revêtira un aspect différent pour chacun : suivre un programme de formation sur l'antiracisme, rapporter les discriminations que l'on observe et défendre la sécurité culturelle autochtone au moyen de l'humilité culturelle. Voilà des mesures importantes permettant de faire une différence significative (https://www.fnha. $\mathrm{ca} /$ wellness/wellness-and-the-first-nations-health-authority/ cultural-safety-and-humility). Les appels à l'action de la Commission de vérité et réconciliation du Canada proposent d'autres voies pour améliorer la qualité des services de santé pour les Autochtones. Notons une meilleure reconnaissance des pratiques de guérison des communautés autochtones et une meilleure représentation des Autochtones au sein de la profession (https://ehprnh2mwo3.exactdn.com/wp-content/ uploads/2021/04/4-Appels_a_l-Action_French.pdf).

Qu'elle soit modeste ou importante, chaque mesure prise contre le racisme systémique est importante. Au cours des mois à venir, la SCPH cessera d'utiliser le terme raciste "Syndrome de l'homme rouge » et rédigera des directives pertinentes portant sur la reconnaissance des termes destinées à la Société. Avec ces efforts apparemment modestes, nous nous engageons à amplifier et à placer la voix des membres des communautés autochtones au centre des débats. Simultanément, nous reconnaissons que les partenaires qui n'appartiennent pas à ces communautés doivent être des instigateurs du changement dans le cadre de leur travail. Nous espérons que vous ferez entendre votre voix et votre expertise auprès de notre communauté. Les membres qui souhaitent contribuer au travail de la $\mathrm{SCPH}$ pour lutter 
contre le racisme sont invités à nous contacter à info@cshp.ca. Nous encourageons particulièrement les personnes ayant vécu une telle expérience à nous contacter si elles se sentent à l'aise de le faire.

En tant que professionnels de la pharmacie, les membres de la SCPH ont l'habitude de faire face à des problèmes complexes, de rechercher des données probantes rigoureuses et de défendre des personnes vulnérabilisées par notre culture. En travaillant ensemble, notre communauté est bien outillée pour lutter pour l'équité, et chacun de nous a un rôle à jouer pour que le racisme dans le système de soins de santé ne soit plus qu'un souvenir.
Jody Ciufo, M. B. A., est directrice générale de la Société canadienne des pharmaciens d'hôpitaux.

Zack Dumont, BSP, ACPR, M. S. (Pharm.), est président et agent de liaison interne de la Société canadienne des pharmaciens d'hôpitaux.

Tania Mysak, B.S.P., Pharm. D., est présidente sortante et agente de liaison pour la vision de la Société canadienne des pharmaciens d'hôpitaux.

Shirin Abadi, B. Sc. (Pharm.), ACPR, Pharm. D., DPLA, MBA, FCSHP, RPh, est présidente élue et agente de liaison externe pour la Société canadienne des pharmaciens d'hôpitaux.

Tamar Koleba, B. Sc. (Pharm.), ACPR, Pharm. D., est Trésorière de la Société canadienne des pharmaciens d'hôpitaux.

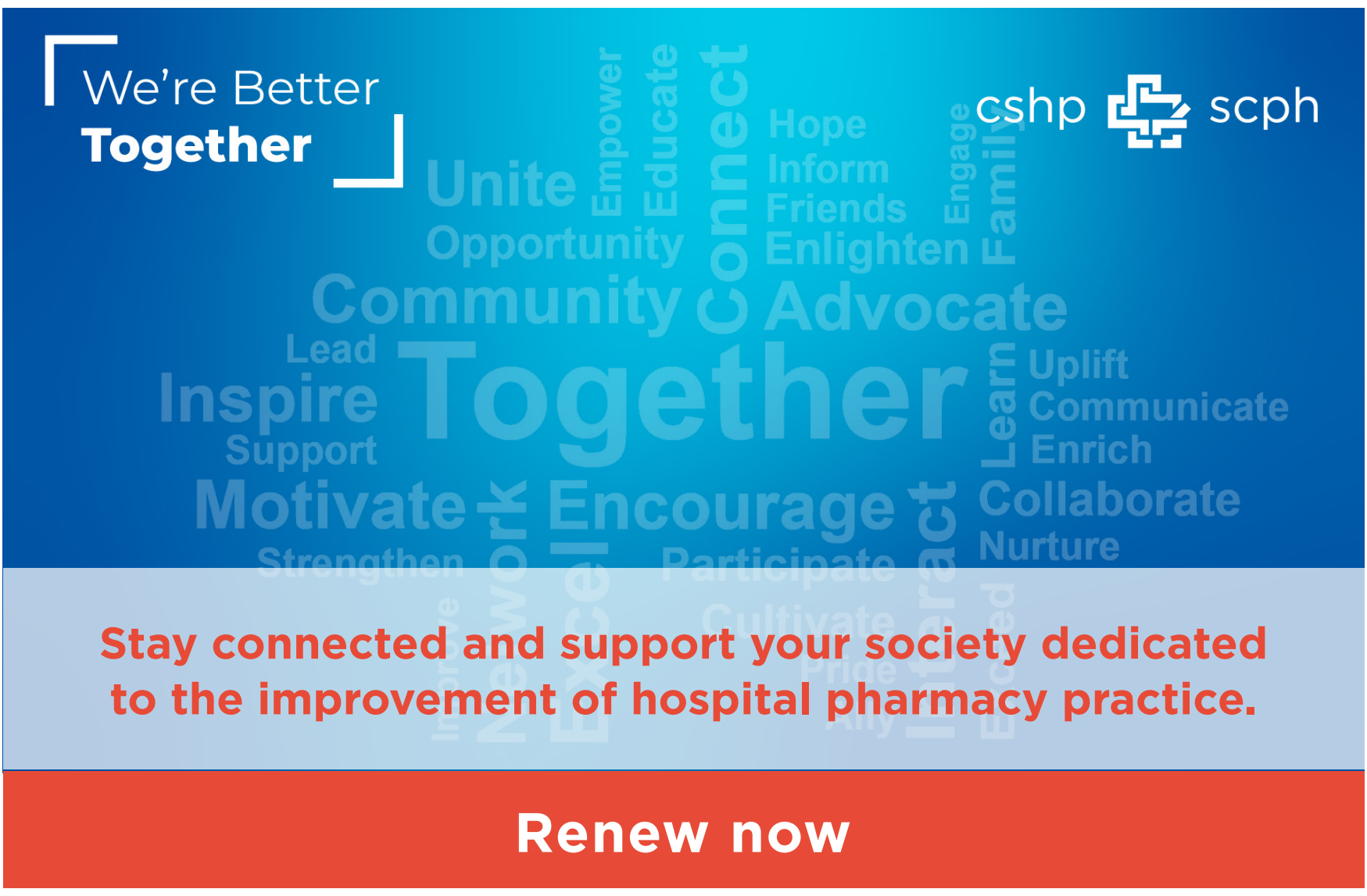

